EPJ Web of Conferences 29, 00007 (2012)

DOI: $10.1051 /$ epjconf/20122900007

(C) Owned by the authors, published by EDP Sciences, 2012

\title{
Performances analysis of a piezo-pump
}

\author{
A. Benaissa, S. Belkhiat ${ }^{\mathrm{a}}$ \\ Laboratoire Dosage Analyse et Caractérisation Haute Résolution, Université Ferhat Abbas Sétif \\ Algérie
}

\begin{abstract}
Piezo-pumps, in the last decade, have received extensive attention because of their potential applications in biomedical devices, biological and chemical analysis, micro-electronics cooling and space exploration. In this paper an analytical model is developed to analysis the parameters effect on the pump flow. The micro-pump driven by piezostack (PZT alloy multilayer) as actuator is studied as function size and working middle. This one is compared with SCMCP pump actuated by PZT alloy as piezomembrane.The flow change as a function frequency, the chamber diameter and control voltage are analyzed. The simulation results obtained with two different liquids (water and blood) as working middle are discussed and compared with experimental results. The results are satisfactory.
\end{abstract}

\section{Introduction}

With the development of micro-electro-mechanical system (MEMS), the piezoelectric pumps have been emerged as a popular area of research. They have received extensive attention because of their potential applications in biomedical devices, biological and chemical analysis, microelectronics cooling, fuel-drop generator for automobile heaters and space exploration [1-3]. Many piezoelectric pumps are developed using piezoelectric membrane actuators (named also piezomembrane pumps) for liquid delivery. They are characterized by their simple structure and small volume. Another type of piezoelectric pump is driven by piezostack actuators which are also called piezhydraulic pumps or piezostack pumps. The two kinds of pump differ point of view power applications. However, the check-valve piezoelectric micropump is particularly appropriate for medical drug or insulin delivery system. A wide bibliography concerning these pumps is given in [4]. The check-valve micropump should be able to provide the precise and repeatable pumped volume of liquid with each cycle, to achieve high flow rate and to void back-flow. For a good control of the liquid delivery system, the mathematical model of the piezopump must be mastered.

In this work the mathematical model for piezostack pump is used as a base [4] combinated to that of connection multi-chamber piezoelectric micropumps (SCMCP). MATLAB/SIMILINK environment is used with the goal of making a simple model that runs on a personal computer. The parameters effect of micropump on the flowrate, especially driving frequency seeing that this one has been few studied in simulation are analyzed. Dimensions, chamber number, pump medium (water and blood) and actuation voltage are considered in the model analysis for optimizing of the

\footnotetext{
a e-mail : belsa_set@yahoo.fr
}

This is an Open Access article distributed under the terms of the Creative Commons Attribution License 2.0, which permits unrestricted use, distribution, and reproduction in any medium, provided the original work is properly cited. 
pump performances. In order to validate the model, the simulation results are compared with the experimental results obtained by others researchers.

\section{Working principle of piezostack pump}

The piezo-pump presented in this paper consists of a piezostack actuator, two check valves and a chamber diaphragm (Fig. 1). When the piezostack actuator is driven by alternating voltage, piezohydraulic pump can transfer mechanical energy into fluid movement. The chamber diaphragm is a flexible membrane with a center-located rigid disk. The radius of the rigid disk and the membrane thickness will exert great effect on pump performance. When the piezostack is operating in extending-shrinking vibration mode, the pressured liquid in pump chamber opens and closes the valve alternately, consequently the liquid moves from the inlet to outlet continuously.

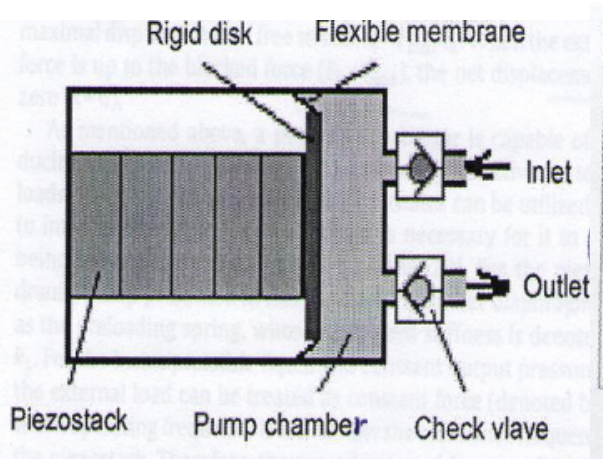

a)

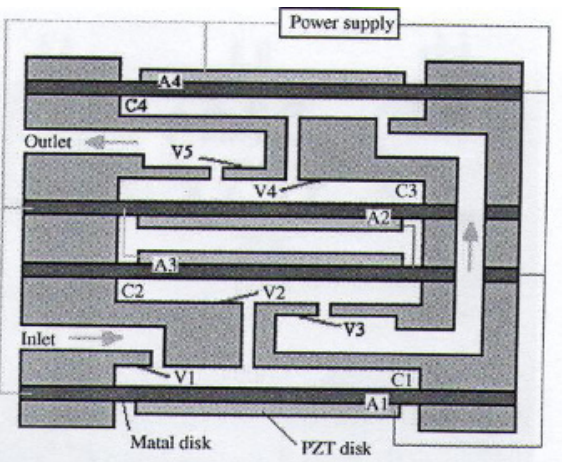

b)

Fig.1Schematic diagram of the pump

a) Piezostack pump b) Serial-connection 4-chamber PZT pump

Then, when the driving frequency is below the resonant frequency of the piezostack actuator, the pump flowrate is expressed by

$$
Q=60 \Delta V \eta_{v} f
$$

Where $\mathrm{f}$ is driving frequency, $\Delta V$ is the volume deflection of pump chamber, $\eta_{v}$ is the check coefficient of valves. $\eta_{v}$ depends on valve type, size and operating frequency. Assuming the thickness of membrane is below that of rigid disk $\left(\mathrm{h}<<\mathrm{h}_{\mathrm{d}}\right)$, the displaced chamber volume per stroke can be calculated by

$$
\Delta V=\pi \frac{x_{0}}{3}\left(a^{2}+a b+b^{2}\right)=\pi \frac{a^{2}}{3} x_{0}\left(1+\lambda_{r}+\lambda_{r}^{2}\right)
$$

where $\mathrm{x}_{0}$ is the maximal displacement of piezostack actuator under liquid pressure $\lambda_{r}=b / \boldsymbol{a}$ is

defined as radius ratio, $a$ and $b$ are the radiuses of the pump chamber and rigid disk respectively.

\section{Analytical model of piezostack pump}

The piezostack is composed of many thin layers of electroactive material alternatively connected to positive and negative terminals of a voltage source. When an electrical voltage is applied, each piezoelectric material layer expands and produces a net output displacement. In the case of both the external force and voltage are applied to the piezoelectric elements, the linear relationship between displacement, force and voltage can be given as [4]:

$$
\mathrm{x}=\frac{1}{\mathrm{k}_{\mathrm{a}}\left(\mathrm{F}_{\text {ind }}-\mathrm{F}\right)}
$$




$$
\begin{aligned}
& F=F_{\text {ind }}-x k_{a} \\
& k_{a}=\frac{A}{l S_{33}^{E}} \\
& F_{\text {ind }}=\frac{1}{t} d_{33} k_{a} V
\end{aligned}
$$

Where $\mathrm{x}$ is the stack displacement, $\mathrm{k}_{\mathrm{a}}$ is the piezostack stiffness, $\mathrm{F}_{\text {ind }}$ is the induced force of the piezostack actuator, $\mathrm{F}$ is the applied external force, $\mathrm{t}$ is the layer thickness, 1 is the stack length, $\mathrm{A}$ is the stack cross-sectional area, $\mathrm{V}$ is the input voltage, $\mathrm{d}_{33}$ is the piezoelectric coupling coefficient between mechanical and electrical variables, $\mathrm{S}_{33}^{\mathrm{E}}$ is the mechanical compliance of the piezoelectric material. In the case of $\mathrm{F}=0$, the applied voltage has as result the maximal displacement of free stroke, $x_{\mathrm{f}}=\mathrm{F}_{\text {ind }} / \mathrm{k}_{\mathrm{a}}$. When the external force is up to the blocked force $\left(\mathrm{F}_{\mathrm{b}}=\mathrm{F}_{\text {ind }}\right)$ the displacement is zero $(\mathrm{x}=0)$.

According to Eq.(4), the maximal displacement of the piezostack under the external load and spring force of chamber diaphragm can be deduced as

$$
X_{0}=\frac{F_{b}-F_{c}}{k_{a}+k_{s}}=X_{f} \frac{1-\lambda_{f}}{1-\lambda_{k}}
$$

where $F_{c}=A_{p} P, \mathrm{~A}_{\mathrm{p}}$ is the cross-section area of the pump chamber and $\lambda_{F}=F_{c} / F_{b}$ is defined as force ratio to denote external load ( liquid pressure), $\lambda_{k}=k_{s} / k_{a}$ is defined as stiffness ratio. In these conditions, the displacement of piezostack increases with the decreasing of force ratio.

$$
x_{0}=\frac{\pi a^{2} x_{f}}{3} \lambda_{V}
$$

Substituting Eq.(9) into Eq. (2) results in:

$$
\Delta V=\frac{\pi a^{2} x_{f}}{3} \frac{\left(1-\lambda_{f}\right)\left(1+\lambda_{r+} \lambda_{r}^{2}\right)}{1+\lambda_{k}}
$$

where $\lambda_{v}$ is defined as the volume coefficient to denote the cability of chamber volume deflection and there is

$$
\begin{aligned}
& \lambda_{V}=\frac{\left(1-\lambda_{f}\right)\left(1+\lambda_{r+} \lambda_{r}^{2}\right)}{1+\lambda_{k}} \\
& \lambda_{f}=\left(1-\lambda_{V}\right) \frac{1+\lambda_{k}}{\left(1+\lambda_{r+} \lambda_{r}^{2}\right)}
\end{aligned}
$$

\subsection{Analytical model of chamber diaphragm}

There is not an available analytical equation to calculated directly the stiffness of a such diaphragm. The authors [4] have treat the chamber diaphragm as an annulus plate inner edge clamed on rigid disk and outer edge on pump body and then establish its deflection function using the summation method presented in [5]. Analytical model of the chamber diaphragm is developed in [4]. We recall here only the stiffness expression, given by

$$
k_{1} s=F_{1} \operatorname{con} /(W(b))=16 \pi D /\left(a^{\uparrow} 2\left[1-\lambda_{1} r^{\uparrow} 2-\left(4 \lambda_{1} r \ln \lambda_{1} r\right)^{\top} 2 /\left(1-\lambda_{1} r^{\uparrow} 2\right]\right)\right.
$$

where $D=\left(E h^{\top} \mathbf{3}\right) /\left[\mathbf{1 2}\left(1-v^{\uparrow} \mathbf{2}\right]\right.$ and $\mathrm{h}$ is thickness of the flexible membrane and $\mathrm{E}$ and $v$ are Young's modulus and Poisson 's ratio, respectively.

For the serial-connection multi-chamber piezoelectric micropumps (SCMCP) [7], the working principle of the diaphragm pump can be described as a periodic process. The output performance is 
equivalent to that of some single-chamber piezoelectric micropump (SCP) operating in series. The backpressure of a SCMCP micropump should be the sum of all chambers running solely such as

$$
\mathrm{P}_{\text {multi }}=\mathrm{P}_{1}+\mathrm{P}_{2}+\cdots, \quad, \mathrm{P}_{\mathrm{n}}
$$

where $\mathrm{n}$ is the chamber number. All chambers share the same check efficiency ( $\eta \mathrm{p})$, then there will be $\mathrm{P}_{\mathrm{i}}=\mathrm{P}_{\mathrm{g}}(\eta \mathrm{p})=\mathrm{P}_{\text {one }}$ and for a SCMCP micropump,

$$
\mathrm{P}_{\text {multi }}=\mathrm{nP}_{\text {one }}
$$

For a SCMCP micropump, the flowrate is expressed in the form

$$
Q=C_{v} A \sqrt{2^{\Delta P} / \rho}
$$

where $\mathrm{C}_{\mathrm{v}}$ is the velocity coefficient, $\mathrm{A}$ the area of the valve orifice, $\rho$ the liquid density, and $\Delta \mathrm{P}$ is the pressure difference from inlet to outlet. When all chambers share the same check efficiency $(\eta \mathrm{Q})$, the flowrate of a SCMCP micropump is expressed as

$$
\mathrm{Q}_{\text {multi }}=\mathrm{C}_{\mathrm{v}} \mathrm{A} \sqrt{\frac{2 \mathrm{nP}_{\text {one }}}{\rho}}=\sqrt{\mathrm{n}} \mathrm{Q}_{\text {one }}
$$

\subsection{Performance of the frequency-dependent flow rate}

The effect of valves on the output performance of the PZT micropump must be mastered for a good control of the pump. The check-valve piezoelectric micropump is appropriate for medical applications such as drug or insulin delivery system. To void the pulsant flow and control the liquid release precisely, a micropump designed for that should be able to operate at a relatively higher frequency.

When the operation frequency is well below the resonant frequency of the PZT actuator, its central displacement can be considered as constant, and the pump volume per stroke $(\Delta \mathrm{V})$ can be calculated as a function the applying voltage [6]

$$
\nabla V=\frac{3 \pi d_{31} U d^{4}}{32 t^{2}}=A_{a} \frac{\pi d^{2}}{8}
$$

where $A_{a}$ is the central displacement of the piezoelectric actuator, $d$ and $t$ are the diameter and thickness of the piezoelectric diaphragm, $d_{31}$ is the piezoelectric constant and $U$ is the applying voltage. The flow rate is then

$$
Q(U, f)=2 \eta \Delta V f
$$

with $\eta$ the check efficency of the valve.

The fluid flow is driven by the vibration actuator to open or close the valves. The actuator vibration, the vave mouvement and the fluid flow are thus coupled. Authors [6] expressed the dynamic equation of the system by a differential equation whose the particular solution is presented as

$$
Y=A_{v} \sin (w t-\varphi)
$$

where $A_{v}$ and $\varphi$ stand for the frequency-dependent amplitude of valve and phase shift which is expressed as

$$
\varphi(w)=\arctan \frac{2 \xi\left(\omega / \omega_{n}\right)}{1-\left(\omega / \omega_{n}\right)^{2}}
$$

where $\xi$ is the damping factor, $\omega$ is the operation pulsation and $\omega_{\mathrm{n}}$ is the actuator pulsation. However, the phase shift increases with the rising of operation frequency and the check efficiency of valve decrease with the increasing of phase shift [6].

$$
\eta=\frac{1}{\varphi}
$$




\section{Simulation results and discussion}

The mathematical model for piezostak pump was used as a base [4]. Piezostack micropumps have been studied in experiments $[4,7]$ and few in simulation. A model that captures the complicated dynamics amenable to control design is important for applying advanced control algorithms to deliver accurate and consistent flow rates for different application. For medical application, the actuation voltage must be low, particularly for the implantable drug-delivery or insulin-delivery system. Recently, a maximum flow rate of $1260 \mu \mathrm{L} / \mathrm{min}$ has been acheived for a polypyrroleactuated micropump under an actuation voltage of $4 \mathrm{~V}$ [8].

The model is so tested on the serial-connection multi-chamber piezoelectric micropumps (Fig.1) [7] whose the piezoelectric diaphragm actuators (PZT alloy as actuators) work in anti-phase, consequently, the output performance is equal to that of several single chamber piezoelectric micropump. This one permits to reach the compromise solution (high flow/low actuation voltage). The excitation voltages are respectively $0-150 \mathrm{~V}$ and $0-40 \mathrm{~V}$ for the two types of pumps. Their frequency games are $0-400 \mathrm{~Hz}$ and $0-600 \mathrm{~Hz}$ and the resonance frequency is equal to $12 \mathrm{kHz}$. The other parameters used for the piezoelectric pump are illustrated in Table 1.

Table 1. Simulation parameters [4]

\begin{tabular}{|c|c|}
\hline \multicolumn{2}{|c|}{$\begin{array}{c}\text { Properties and dimensions of chamber diaphragm (beryllium } \\
\text { bronze) }\end{array}$} \\
\hline Youngs modulus $\mathrm{E}_{\mathrm{m}(\mathrm{GPa})}$ & 125 \\
\hline Poisson ratio $v$ & 0.35 \\
\hline Membrane thickness h (mm) & 0.2 \\
\hline Rigid disk thickness $h_{d}(\mathrm{~mm})$ & 3 \\
\hline Membrane radius a (mm) & 15 \\
\hline Density $\rho\left(\mathrm{kg} / \mathrm{m}^{3}\right)$ & 8920 \\
\hline \multicolumn{2}{|c|}{ Properties and dimensions of piezostack } \\
\hline Piezostack type & PSt $150 / 5 / 80 \mathrm{VS} 10$ \\
\hline Maximum displacement $\mathrm{x}_{\mathrm{f}}(\mu \mathrm{m})$ & 80 \\
\hline Voltage range $(\mathrm{V})$ & $0-150 ; 0-40$ \\
\hline Stack stiffness $\mathrm{k}_{\mathrm{a}}(\mathrm{kN} / \mathrm{mm})$ & 6 \\
\hline Length $(\mathrm{mm})$ & 82 \\
\hline Blocked force $\mathrm{f}_{\mathrm{b}}(\mathrm{N})$ & 800 \\
\hline Capacitance C (nF) & 3200 \\
\hline Resonance frequency $(\mathrm{kHz})$ & 12 \\
\hline
\end{tabular}

There are many factors exert influence on the piezostack pump sch as driving frequency, stack size, thickness of chamber diaphragm, radius of disk valve type as well as liquid properties. This work focuses only the effect of the disk radius and the driving frequency on the flowrate of pumps seeing that other parameters effect has been simulated in the literature.

\subsection{Radius effect on flowrate}

Fig. 2 shows the influence of the frequency driving on flowrate of piezostack pumps. The flowrate has been simulated for four values of radius $(8 \mathrm{~mm}, 10 \mathrm{~mm}, 12 \mathrm{~mm}$ and $14 \mathrm{~mm})$. The simulation results are presented in Fig. 2,b. The simulation results are close to experimental resultat as well as in form and in amplitude. The frequency corresponding to highest flowrate is same to that of experimental curve. It is located between $270 \mathrm{~Hz}$ and $300 \mathrm{~Hz}$ for the four value of radius.

However, we remark that in the simulation curves, the maximum flowrate for $r=8 \mathrm{~mm}$ and $r=10$ $\mathrm{mm}$ is smaller than experimental results. They are respectivement equal to $150 \mathrm{ml} / \mathrm{mn}$ and 200 $\mathrm{ml} / \mathrm{mn}$. These values are respectively 0.6 and 0.74 time smaller than experimental maximum. However the highest flowrate corresponding to $\mathrm{r}=12 \mathrm{~mm}$ and $14 \mathrm{~mm}$ are equal to experimental measures. Simulation and experimental results show that the optimum radius for an optimum flowrate is $r=12 \mathrm{~mm}$. 

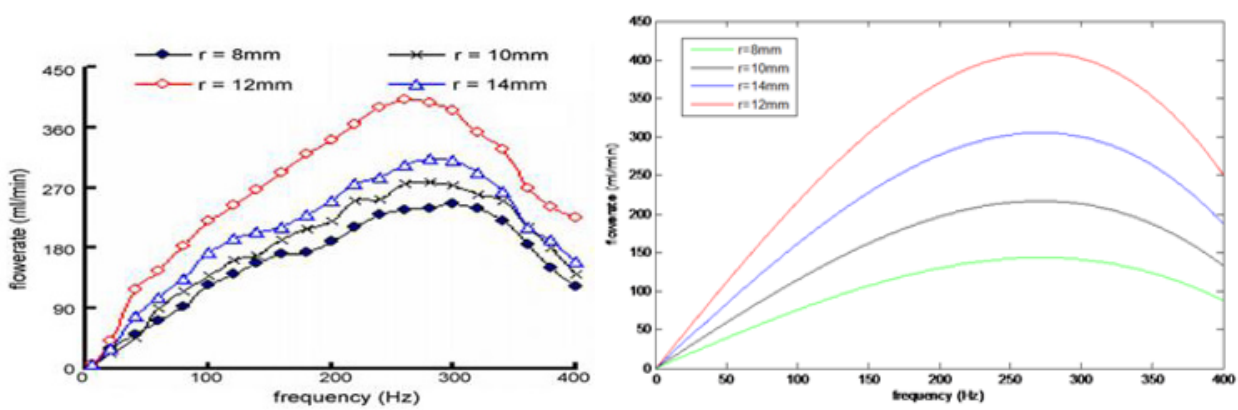

Fig. 2. Influence of driving frequency on flowrate of piezostack (water as working middle) a) experimental measurement [4], b) simulation result

\subsection{Actuation voltage effect}

The actuation voltage is illustrated in the figure 3 . The result shows that flowrate is in direct proportion to actuation voltage. Highest flowrate is reached at 150 volts for the piezostack pump.

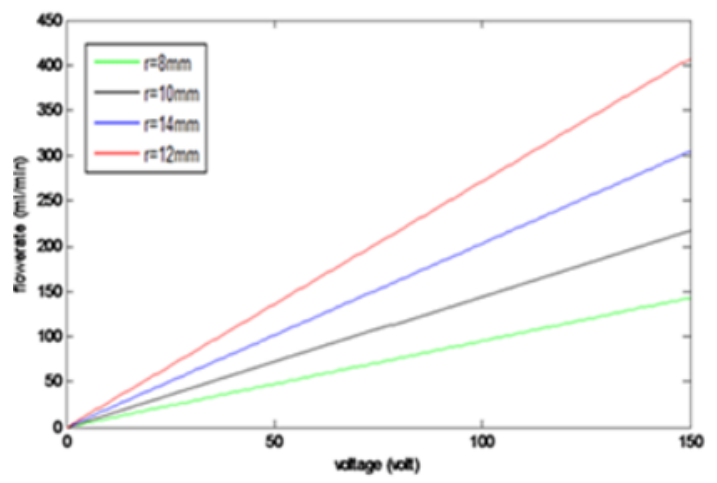

Fig. 3. Flowrate as a function actuation voltage

The influence of driving frequency on flowrate for different values of actuation voltage $(5 \mathrm{~V} ; 10 ; 25 \mathrm{~V}$ and $40 \mathrm{~V}$ ) for a piezostack pump is represented in the figure 4.

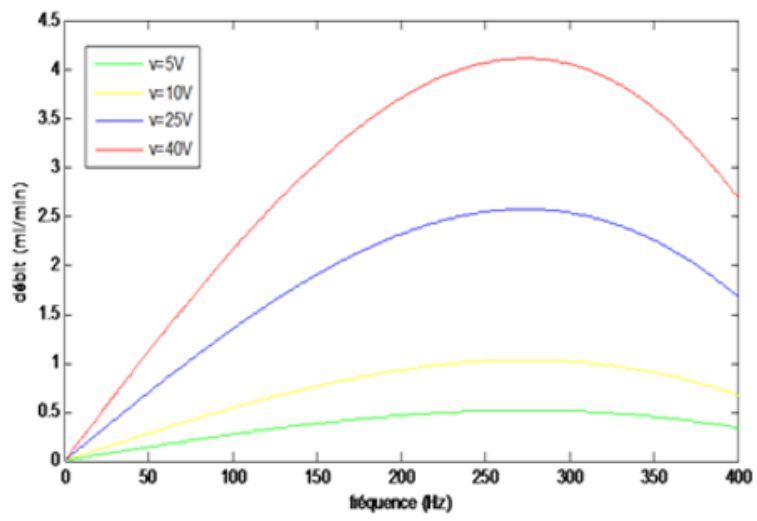

Fig. 4. Influence of driving frequency on flowrate for different values of actuation voltage: $5 \mathrm{~V} ; 10 ; 25 \mathrm{~V}$ and $40 \mathrm{~V}$ for a piezostack pump 
For to reach a maximum flowrate of $1260 \mu \mathrm{L} / \mathrm{min}$ (or more) acheived for a polypyrrole-actuated micropump under an actuation voltage of $4 \mathrm{~V}$ [8], piezostack pump must be actuated with a voltage more than $10 \mathrm{~V}$. Fig. 4 shows that piezostack pump actuated with $25 \mathrm{~V}$ can be suitable for medical application since the maximum flowrate is greater than $1.5 \mathrm{ml} / \mathrm{mn}$.

\subsection{Simulation of serial-connection multi-chamber pump}

For increase the flowrate using a low actuation voltage serial-connection multi-chamber pump (SCMCP) has been simulated (Fig. 5). The actuation voltage is 40V. The evolution of relationship between the flowrate and driving frequency for four chambers is given. Each curve represents the number of serial-connection chamber. The simulation results are in good agreement with experimental result. A little difference is observed only on the increasing part of the simulation curve representing the serial-connection of four chambers. The increasing part of the curve is shifted towards the high frequency. However, maximum flowrate and maximum driving frequency increase with the chamber number in serial-connection.

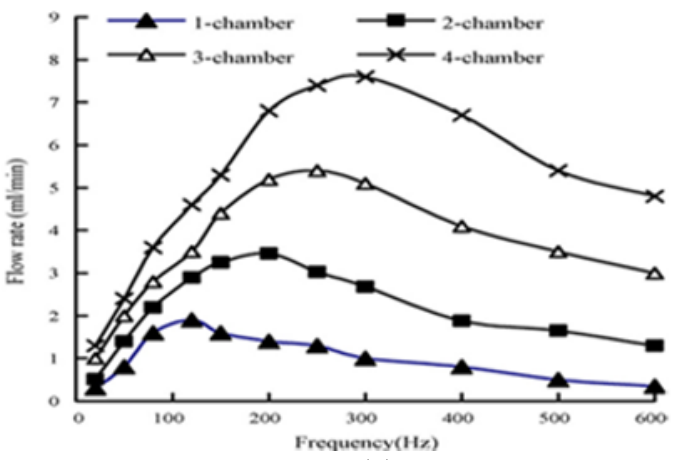

(a)

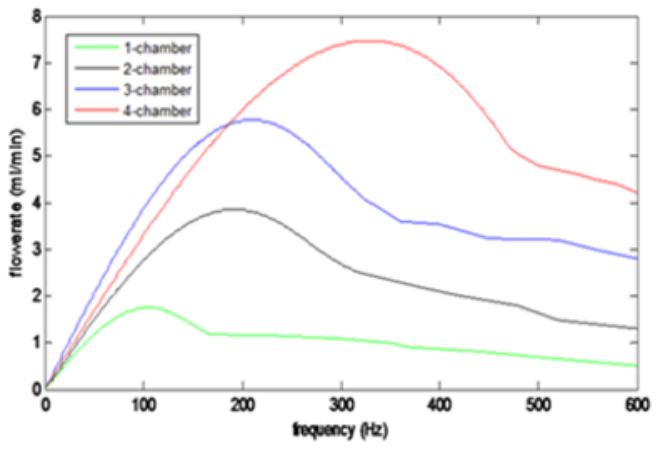

(b)

Fig. 5. The relationship between the flowrate and driving frequency; water as working middle a) experimental results [7] / b) Simulation results

Fig. 4 (curve 40V) and Fig. 5 (curve 4-chamber) shows well the performances of SCMCP in comparison with piezostack pump. The maximum flowrate of SCMCP (at the same driving frequency) is two times greater than that of piezostack pump.

\subsection{Effect of the medium pump on the flowrate}

As all systems possessing mass and elasticity, the valve is capable of free vibration in liquid. We must take account the liquid density and the liquid dynamic viscosity which depend of liquid type.
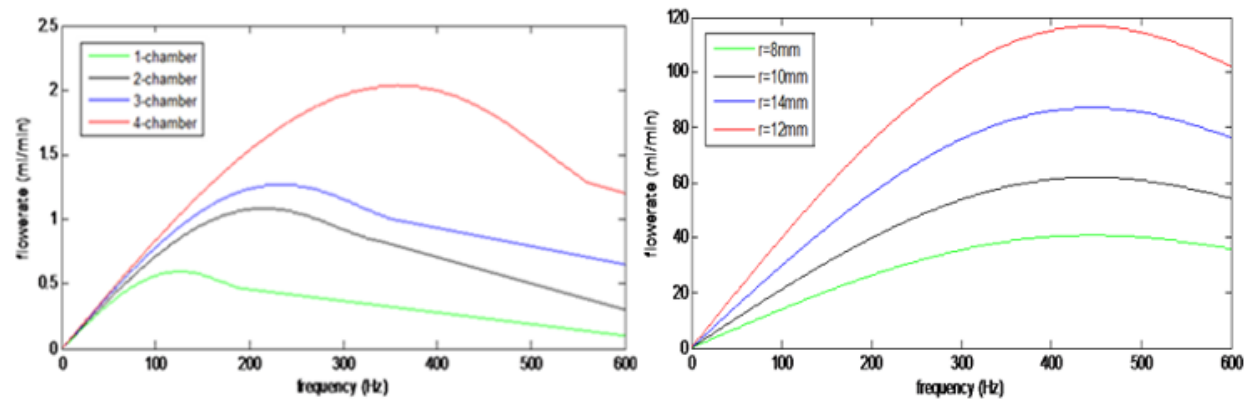

Fig. 6. The relationship between the flowrate and driving frequency; blood as working middle a) Simulation results, SCMCP pump

b) Simulation results,piezostack pump. 
Previously, water has been considered as working middle for to compare the results permitting to validate the model. We show in this section the effect of the pump middle on the flowrate. Human blood as working middle is simulated in Fig. 6.

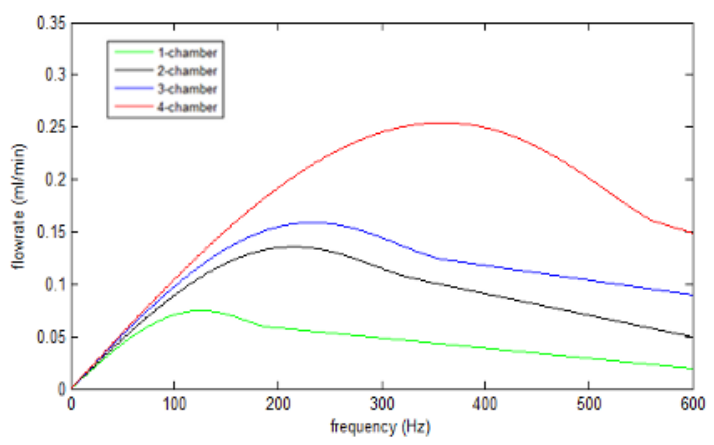

Fig. 7. Simulation results, SCMCP pump with $5 \mathrm{~V}$ as actuation voltage

Fig. 6, a and 6, b show respectively the relationship between the flowrate and driving frequency where blood is the working middle of SCMCP pump and of piezostack pump. In the two cases of pump, the maximum flowrate has decreased 3.5 times in comparison with that results obtained with water as pump middle (see Fig. 2 and Fig. 5). The maximum driving frequency shifted between 350$400 \mathrm{~Hz}$ (Fig. 6,a) and 400-450 Hz (Fig. 6,b). These results show well the effect of liquid density and liquid dynamic viscosity on the flowrate, driving frequency, and confirm also their dependence with the valve frequency (see [6] Eq.(7)).

However, Flowrate delivery by SCMCP pump under $5 \mathrm{~V}$ as actuation voltage is presented in Figure 7. The value of maximum driving frequency for each chamber in serial-connection is the same that one obtained with an actuation voltage equals to $40 \mathrm{~V}$ (Fig.6,a) but the flowrate is ten times smaller. Maximum flowrate delivered by 4-chamber is $0.25 \mathrm{ml} / \mathrm{mn}$ (Fig. 7). In comparison with piezopump based electroactive polymer as piezomembrane (actuation voltage 4V [8]), the flowrate is $(1.26 / 0.25)$ five times smaller. This result shows that electroactive polymer as piezomembrane provides a compromise solution high flowrate (more than $1.25 \mathrm{ml} / \mathrm{mn}$ ) with low actuation voltage $(5 \mathrm{~V})$.

From the study carried out, we can say, the piezoelectric pump is a complex system nonlinear where many factors exert effect on the flowrate such as working parameters (voltage and frequency) and geometrical parameters of the PZT actuator. The choice of type pump and of voltage actuation is determinate by the application of the piezopump. PZT alloy multilayer (called piezostack) is recommended so for to delivery high flowrate with high voltage actuation. However, SCMCP pump or piezoelectric membrane (PZT alloy) pump can satisfy the need of some medical applications where the voltage actuation must be lower. For implantable system, see [8], micropump based on electro active polymer actuation, is recommended.

\section{Conclusions}

The background in the field of piezopump modeling has been discussed in this paper. A mathematical description of piezostack and SCMCP pump is developed. The analytical equation derived above allows one to predict the performance of piezo-pump. It has well been showed that many factors exert influence on piezo-pump, such as driving frequency, dimensions piezo-pump components, thickness of layer or multilayer of piezoelectric material, valve effect, voltage actuation as well as liquid properties. This paper focus only on the influence of driving frequency on flowrate, voltage actuation and working middle simulation seeing that the effect of others parameters has been simulated in the literature. The comparison between the experimental and simulated results shows a good agreement. A qualitative validation of the results obtained is provided by Junwu Kan 
$[4,7]$. Finally, validation of the extended model was performed; we notice that the small difference between measurement and simulation results originates from the dynamic of the valve in the working middle. The choice of pump type depends of application field. In the next work, the model will be tested on the piezo-polymer.

\section{References}

1. P. Woias, Pums-past, progress and future prospects, Sens. Actuators B 105 28-38 (2005).

2. W. J. Spencer, W.T. Corbett, L.R. Dominguez, et al., An electronically controlled piezoelectric insulin pump and valves, IEEE Trans. Son. Ultrason. 3153-156, SU-25 (1978).

3. A. Nisar, N. Afzulpurkar, B. Mahaisavariya, A. Tuantranont, MEMS-based micropumps in drug delivery and biomedical applications, Sensors and Actuators B130917-942 (2008).

4. Junwu Kan, Kehong Tang, Yu Ren, Guoren Zhu, Peng Li, Study on a piezohydraulic pump for linear actuators, Sensors and Actuators A, 149 331-338 (2008).

5. F. He, Y. Shen, Theory of plate and shells, First edition, Xi'an Jiaotong University, Xi'an (1993).

6. Kan Junwu, Yang Zhigang, Peng Taijiang, Cheng Guangming, Wu Boda, Design and test of a high-performance piezoelectric pump for drug delivery, Sensors and actuators A 121 (2005)156161

7. Junwu Kan, Kehong Tang, Guojun Liu, Guoren Zhu and Chenghui Shao, Development of serialconnection piezoelectric pumps, Sensors and Actuators A 144 321-327 (2008).

8. Yang Fang, Xiaobo Tan, A novel diaphragm micropump actuated by conjugated polymer petals: Fabrication, modeling, and experimental results, Sensors and Actuators A 158 121-131 (2010). 